\title{
A Novel Infrared Detector Using Highly Nonlinear Twisting Vibration
}

Tatsuya Yamazaki, Shouhei Ogawa, Shinya Kumagai, Minoru Sasaki

Department of Advanced Science and Technology, Toyota Technological Institute,

Nagoya 468-8511, Japan

\section{Corresponding Author:}

Minoru Sasaki

Professor

Department of Advanced Science \& Technology

Toyota Technological Institute

2-12-1 Hisakata, Tempaku-ku, Nagoya 468-8511, Japan.

E-mail: mnr-sasaki@toyota-ti.ac.jp

Phone: +81-52-809-1840

Fax: +81-52-809-1721 


\begin{abstract}
A new resonant infrared (IR) detector is proposed. The twisting motion of the thin film torsion bar is first used. The resonant frequency gives the information of the temperature change of the moving element caused by IR absorption. The dynamic sensing using the electrostatic force can remove the drift without the heat generation. The large nonlinearity of the thin film torsion bar combined with the frequency measurement increases the advantage of the high sensitivity. The IR detector is fabricated by the surface micromachining using sub- $\mu$ m thick poly-Si film. Its structure consists of two parts. One is the thin film torsion bars and another is the bending center part. They can be basically designed separately. The center part has bi-layer structure to induce bending against the temperature increase caused by the IR absorption. One fabricated IR detector has the resonant frequency of about $35 \mathrm{kHz}$ and shows the sensitivity of $30 \mathrm{~Hz} / \mathrm{K}$ and the shifting ratio of $830 \mathrm{ppm} / \mathrm{K}$. This sensitivity is larger than the previously reported Si detectors. The shifting ratio is larger than devices including the high-frequency quartz and $\mathrm{ZnO}$ detectors.
\end{abstract}

\title{
Keywords:
}

resonant IR detector; twisting vibration; nonlinear spring; thin film torsion bar; high sensitivity 


\section{INTRODUCTION}

Sensitive infrared (IR) detection is needed in many fields. The potential applications are human sensing, security, power-saving control, molecular detection, environmental monitoring, and so on. However, there is no suitable IR detector. As the visible light for comparison, the detectors are well-developed and the imaging devices are installed in many products which are frequently portable working with the battery power. The essential reason of the delay of the technological development in IR region is the fact that IR photon has smaller energy compared to the visible one. The handling of smaller energy needs the more delicate technique. Generally, there are two types of IR detectors. One is photon type detector, which transduces the incident IR photon into the electric charge using semiconductor materials. Photodiodes or many image sensors for visible light are belonging to this. Historically, the photon detectors have been preferred for the higher sensitivity. The band gap has to be narrow for matching with IR energy[1]. Sensitivity can be enhanced by incorporating quantum dots[2]. The detectors need cooling since they are bothered by the noise due to the thermally excited electric carriers. So, the photon detector is not suited for low power consumption and for portable equipments. As for IR having the relatively short wavelength, the plasmonic excitation and the detection of the hot electron is the recent trial[3].

From the view point of the applications, the thermal type detector is attractive since the cooling becomes unnecessary. The recent advances in micromachining techniques improve the thermal detectors[4]. When the incident IR is absorbed, the local heating in the element occurs and the resultant higher temperature generates subsequent changes. The suspended microstructure improves the thermal isolation making the temperature increase $\Delta T$ larger. Various mechanisms are proposed for the thermal detectors. The typical devices are bolometers[5], thermopiles[6-9], bimetals[10-14], and diodes[15,16]. In addition to the above static detectors, the resonant IR detectors are investigated. The potentially higher sensitivity can be achieved because the frequency shift can be measured with high accuracy. The bending-type Si-based bridge and cantilever have been reported [17, 18]. When 
$\mathrm{Si}$ is used as the structure material, the temperature sensitivity of Young's modulus is only about $-35 \mathrm{ppm} / \mathrm{K}$ [19-21]. So, the simple Si structure is not sensitive. They introduce the structures for accumulating the thermal stress inside. The bending of the thin film is influenced by the heat conduction and thermal stress to generate the frequency change. The mechanisms used are essentially unclear and complex having the difficulty in the control and the stable operation. Other resonant IR detectors have been tried using Y-cut quartz resonator [22], and $\mathrm{ZnO}$ based film acoustic resonator[23]. They use the large resonant frequency over MHz. The resultant frequency shift becomes large although the ratio is similar value. Y-cur quartz resonator has the resonant frequency of $90 \mathrm{MHz}$, and gives the sensitivity of $7.2 \mathrm{kHz} / \mathrm{K}$ or $80 \mathrm{ppm} / \mathrm{K}$ in the ratio. $\mathrm{ZnO}$ resonators have the resonant frequency of $1.82 \mathrm{GHz}$, and the sensitivity of $-73 \mathrm{ppm} / \mathrm{K}$ in the ratio. The sensing bases on the temperature dependence of the material used. The materials of the quartz or $\mathrm{ZnO}$ are not well-matched with CMOS devices. There is also the difficulty for combining with the suspended microstructure for obtaining the thermal isolation and the larger temperature increase $\Delta T$.

In this study, a new thermal type infrared detector is proposed based on the nonlinearity of the twisting spring constant of the thin film torsion bar. The twisting vibration is useful for avoiding the buckling instability. In this mechanical system, the twisting and the bending motions are coupled for obtaining the nonlinear effect. The devices can be fabricated based on the poly-Si surface micromachining having the good compatibility with CMOS circuits and arraying.

\section{PRINCIPLE AND BASIC POLICY}

Figure 1(a) illustrates the schematic drawing of the detector proposed. A torsional resonator is made from the tense thin film of the poly-Si. Figure 1(b) shows the condition that the twisting element is straight. The center part has two layers. The top layer is the material (metal) having the larger thermal expansion ratio compared to the bottom poly-Si layer. When the incident IR is absorbed, the resonator is designed to bend upwards due to the larger thermal expansion of the layered structure at the center as shown in Fig. 1(c). This bending makes the torsion bar slant against 
the line connecting the fixed ends and hardens the torsional spring. The resonant frequency increases as the result. IR sensing is measuring this increase. The frequency measurement is suited for obtaining the high resolution and for reducing the drift. The electrostatic force is used for exciting twisting since this does not generate the heat without disturbing the detection of the local temperature increase generated by the IR absorption.

The hard spring effect of the torsion bar is originally observed in the micromirror developed by our group[24]. The resonant frequency is observed to increase from 360 to $670 \mathrm{~Hz}$. This corresponds to the increase of spring constant more than 3-times. This phenomenon is analyzed and compared with the experiments[24, 25]. Here, the phenomenon is reconsidered introducing a new factor of swinging. Counting the shear stress, the torsional spring constant has the well-known formula.

$$
k_{\text {shear }} \approx \frac{2 G w t^{3}}{3 l}\left(1-\frac{192}{\pi^{5}} \frac{t}{w} \tanh \frac{\pi w}{2 t}\right)
$$

This is for two torsion bars. $G, w, t$, and $l$ are the torsional rigidity, the width, the thickness, and the length of one torsion bar, respectively. For evaluating the torsional spring constant in this proposed device, the stretching and bending factors have to be included in addition to the shear stress because the device is doubly-clamped requiring the significant force for its deformation. The torsion bar can be modeled as shown in Fig.2. When the film has the residual stress, the stretching factor can be larger since this is the surface force maintaining its magnitude in the small dimension compared with the bulk force. This twisting spring constant is evaluated based on the energy method[26]. For clearing the design guideline, the model is reconsidered here. Along the torsion bar, $x$-axis is set placing the origin at its center. The center bending part of the detector device shown in Fig. 1 is omitted connecting two torsion bars. The trial function of the bending profile $\widehat{\omega}_{c}(x)$ in $z$-direction is approximated as a form of cosine.

$$
\widehat{\omega}_{c}(x)=\frac{z_{m}}{2}\left(1+\cos \frac{2 \pi x}{L}\right)
$$

The displacement of the torsion bar in $x y z$ directions are expressed as $u v w$, respectively. The 
subscript $c$ indicates the center of cross-section of the torsion bar at position $x$. The bending height $z_{m}$ increases with IR absorption. $L=2 l$ is the full length of two torsion bars. Here, the twisting is supposed to distribute uniformly supposing the trial function of the twist angle $\hat{\theta}(x)$ as follows.

$$
\hat{\theta}(x)=\theta_{0}\left(1-\frac{2}{L}|x|\right)
$$

$\theta_{0}$ is the twist angle at $x=0$. Twisting makes the cross-section slant against the original horizontal surface. The twisting is simply expressed as follows.

$$
\widehat{\omega}(x)=\widehat{\omega}_{c}(x)+\xi \hat{\theta}(x)
$$

In the cross-section $S, \xi \eta$-axes are set as shown in the inset of Fig. $2(-w / 2<\xi<w / 2,-t / 2<\eta<t / 2)$. Accompanying the bending and the twisting, the torsion bar will swing around the fixed points. The shift in $y$-direction occurs. The trial function is supposed to be as follows.

$$
\widehat{v}(x)=-\widehat{\omega}_{c}(x) \hat{\theta}(x)
$$

This swinging $\hat{v}(x)$ is newly counted. $\widehat{\omega}_{c}(x)$ is used as the approximation. When $\hat{\omega}(x)$ is used, the result cannot be expressed by the simple polynomial. The length change of the torsion bars is given by as follows.

$$
\delta L=\int_{-L / 2}^{L / 2}\left\{\frac{1}{2}\left(\frac{d \widehat{w}}{d x}\right)^{2}+\frac{1}{2}\left(\frac{d \widehat{v}}{d x}\right)^{2}\right\} d x
$$

The strain energy $W_{r}$ worked against the residual film stress $\sigma_{0}$ (or tension inside the torsion bar) is obtained by the integration over the cross-section $S$ and the length.

$$
W_{r}=\sigma_{0} \int_{S} d S \int_{-L / 2}^{L / 2}\left\{\frac{1}{2}\left(\frac{d \widehat{w}}{d x}\right)^{2}+\frac{1}{2}\left(\frac{d \widehat{v}}{d x}\right)^{2}\right\} d x
$$

The torque generated from the film stress is obtained by the differentiation with respect to $\theta_{0}$.

$$
T_{r}=\frac{\partial W_{r}}{\partial \theta_{c}}=\frac{\sigma_{0} w t}{L}\left\{\frac{w^{2}}{3}+\left(\frac{\pi^{2}}{6}+\frac{15}{4}\right) z_{m}^{2}\right\} \theta_{0}=k_{r} \theta_{0}
$$

The spring constant $k_{r}$ can be expressed as follows. 


$$
k_{r}=\frac{\sigma_{0} w t}{L}\left\{\frac{w^{2}}{3}+\left(\frac{\pi^{2}}{6}+\frac{15}{4}\right) z_{m}^{2}\right\}
$$

Although $\sigma_{0}$ is usually less than $1 \%$ of $G, k_{r}$ can be the significant magnitude, since $k_{\text {shear }}$ decreases by $t^{3}$ with smaller $t$, but $k_{r}$ decreases by $t$. The total spring constant is the sum.

$$
k_{\text {total }}=k_{\text {shear }}+k_{r}+k_{\text {bend }+ \text { stretch }}
$$

$k_{\text {bend }+ \text { stretch }}$ counts the strain (bending and stretching) energy being proportional to Young's modulus.

$$
W_{\text {bend }+ \text { stretch }}=\frac{E}{2} \int_{S} d S \int_{-L / 2}^{L / 2}\left\{-z \frac{d^{2} \widehat{w}}{d x^{2}}+\frac{1}{2}\left(\frac{d \widehat{w}}{d x}\right)^{2}+\frac{1}{2}\left(\frac{d \widehat{v}}{d x}\right)^{2}\right\} d x
$$

This results in the following spring constant using the similar calculation.

$$
k_{\text {bend }+ \text { stretch }}=\frac{E w t}{2 L^{3}}\left\{\frac{w^{2}}{3}+\left(\frac{\pi^{2}}{6}+\frac{15}{4}\right) z_{m}{ }^{2}\right\}\left\{\frac{w^{2} \theta_{0}{ }^{2}}{3}+\left(\frac{\pi^{2}\left(3+\theta_{0}{ }^{2}\right)}{6}+\frac{15 \theta_{0}{ }^{2}}{4}\right) z_{m}{ }^{2}\right\}
$$

This value will be relatively smaller, since the small factors of $w / L$ and $z_{m} / L<<1$ are multiplied additionally. Supposing one design values shown in the caption of Fig. 2, which corresponds to one device design described later, the calculated values for $k_{\text {shear }}, k_{r}$, and $k_{\text {bend }+ \text { stretch }}$ are $6.7 \times 10^{-10}$, $2.9 \times 10^{-10}$, and $9.2 \times 10^{-11} \mathrm{Nm} / \mathrm{rad}$, respectively. Supposing $z_{m}=2 t$, the nonlinear second term in $k_{r}$ Eq.(9) is $8.6 \times 10^{-11} \mathrm{Nm} / \mathrm{rad}$, and the terms including $z_{m}{ }^{2}$ in $k_{\text {bend }}$ stretch Eq.(12) is $9.0 \times 10^{-11} \mathrm{Nm} / \mathrm{rad}$. Their total corresponds to $17 \%$ against the total $k_{\text {total }}$. The term including $z_{m}{ }^{2}$ in Eq.(9) is from the swing factor $v$. Compared with the first term having $w^{2}$, this second term becomes larger when $z_{m} / w>0.249$. This gives $z_{m}>1.25 \mu \mathrm{m}$ when $w=5 \mu \mathrm{m}$. Although the actual $w$ can be smaller than $5 \mu \mathrm{m}$ due to the over-etching of $\mathrm{Si}$, the non-linear effect is clear in experiment even when $z_{m}$ is smaller. The possible reason of the under-estimation of the nonlinearity in the above calculation is the fact that Eq.(3) supposes the uniform distribution of twist angle. With bending $z_{m}$, the twisting region in the torsion bar can decrease. This will decrease the effective length $(l$ and $L)$ increasing all spring constants in Eq.(10).

The resonant frequency of the torsional vibration is given by 


$$
f_{r}=\frac{1}{2 \pi} \sqrt{\frac{k_{\text {total }}}{I}}
$$

where $I$ is the moment of inertia of the moving element. Although the magnitude of $I$ changes when the rotation axis shifts with the bending of the torsion bar, this change is relatively small because the moving element is designed to be larger than $z_{m}$. With larger $z_{m}$, the changing ratio of $k_{\text {total }}$ increases giving the higher sensitivity. The thermal bending of the resonator used in the principle is static, and the twisting motion is dynamic. The change of the resonant frequency can be obtained even when the twisting angle $\theta_{0}$ is small. This is different from the usual nonlinearity depending only on $\theta_{0}$.

As the basic policy of the device design, the torsion bar should be as thin as possible. This will increase $z_{m}$. The width $w$ is better to be narrower for obtaining the nonlinear effect. Also for decreasing the thermal conduction, the thinner and narrower torsion bar is advantageous. For generating the larger bending height $z_{m}$, the center part to absorb IR is better to be larger. So, the device tends to be larger at the center part supported by the weaker suspension of the thin film torsion bar. There will be the trade-off for realizing the device in the fabrication process.

\section{FABRICATION}

Figure 3 shows the fabrication sequence based on the surface micromachining showing A-A' cross-section. The substrate is highly doped n-type $\mathrm{Si}$ and thermally oxidized. The obtained $3 \mu \mathrm{m}$-thick $\mathrm{SiO}_{2}$ layer is used as the sacrificial layer later. An amorphous Si film (thickness: 400nm) is deposited by the low-pressure chemical vapor deposition. After the deposition without doping, B ions are implanted for obtaining the electric conduction. The amorphous $\mathrm{Si}$ film is annealed at $800^{\circ} \mathrm{C}$ for $5 \mathrm{~h}$ to generate the crystallization-induced tensile stress in the resultant poly-Si film [27] and activate the implanted B (step 1). This poly-Si film is patterned (step 2) and plasma etched (step 3). The underlying sacrificial $\mathrm{SiO}_{2}$ layer is wet-etched using $\mathrm{HF}$ solution. The sample is treated by the supercritical drying to suppress the stiction (step 4). Au/Cr layers (thickness: 200/20nm) for making bi-materials structure at center and electrode pads are deposited through the stencil mask (step 5). 
This is for avoiding the electrochemical etching around the $\mathrm{Au} / \mathrm{Cr}$ patterns. When $\mathrm{Au} / \mathrm{Cr}$ layers are deposited before HF etching, the apparent damage of poly-Si or deformation on $\mathrm{Au}$ area are frequently observed degrading the fabrication yield to be about $30 \%$. Au/Cr deposition after the sacrificial etching as shown in Fig. 3 gives the yield to be about $90 \%$. IR absorbing material is not deposited in this study for the fabrication simplicity. Since the total yield obtaining the driving performance is not high at present, the measurement is carried out using the working devices obtained. For larger bending height $z_{m}$, the movable structure is prepared from the thinner film. In case of polyMUMPs of 3-layer poly-Si surface micromachining, the releasable poly-Si layers are 1.5 or $2.0 \mu \mathrm{m}$ thick[28]. So, 400nm-thick poly-Si film used in this study is thinner. The structure is significantly soft against bending. This makes the fabrication process difficult.

Figure 4(a) shows one device prepared using the stencil mask. Arrayed resonators are connected with the same driving electrode. The protrusion at the center having the size of $5 \times 5 \mu \mathrm{m}^{2}$ is for decreasing the stiction. The obtained alignment accuracy between the structure and the stencil mask is about $100 \mu \mathrm{m}$. This device has the torsion bar having $5 \mu \mathrm{m}$-wide and $20 \mu \mathrm{m}$-long. The both end has the chamfering with $5 \mu \mathrm{m}$-side for avoiding the stress focusing. The center moving part is $25 \mu \mathrm{m}$-wide and $200 \mu \mathrm{m}$-long. Figure 4(b) shows another design prepared with Au/Cr patterning before HF etching although the yield is low. The dimension difference is the torsion bar length of $40 \mu \mathrm{m}$. The center moving part is $40 \mu \mathrm{m}$-wide and $400 \mu \mathrm{m}$-long. Figure $4(\mathrm{c})$ shows the magnified view around the torsion bar. The initial bending can be seen. The connection point with the torsion bar has $5 \mu \mathrm{m}$-offset from the center. Some design variation is prepared. As for the torsion bar, the width is $5 \mu \mathrm{m}$, the length is $20,40,90$, and $190 \mu \mathrm{m}$. As for the center part for bending, the width is 25 , $40,60 \mu \mathrm{m}$, the length is 100,200 , and $400 \mu \mathrm{m}$. The connecting point with the torsion bar has the offsets from the center when the center moving part is wider than $40 \mu \mathrm{m}$. Since the gap between the resonator element and the substrate is $3 \mu \mathrm{m}$, the mechanical twist angle allowed is less than $7^{\mathrm{O}}$. The higher yield is observed for the devices with the relatively harder springs. 


\section{STATIC PERFORMANCE}

The thermal bending of the resonator is measured using the white light interferometer (Zygo, NewView 7300) in air. Figure 5(a) shows the experimental setup. The IR detector is heated from the substrate side using the hot plate. The temperature is measured on the hot plate although the actual bending due to the IR irradiation can be different in the exact meaning. Figure 5(b) shows the top surface profile including $\mathrm{Au} / \mathrm{Cr}$ layer not the profile of poly-Si film. One of the reasons of the unsymmetrical profile is the deposited material under the low alignment accuracy of the stencil mask. Since the resonator is $400 \mathrm{~nm}$-thick and $500 \mu \mathrm{m}$-long, its profile easily deflects. The stress during the processes from the wet etching to drying is considered to be large resulting in the plastic deformation. Evaporated $\mathrm{Au} / \mathrm{Cr}$ layer is known to have the tensile stress[29], which can bend the center part downward although the poly-Si film also has the tensile stress inside. Figure 5(b) shows the profiles of the detector at the temperature of $30,110,150^{\circ} \mathrm{C}$. When the temperature increases from 30 to $150^{\circ} \mathrm{C}$, the center part of the IR detector shits upwards. At $150^{\circ} \mathrm{C}$, this bending height reaches $400 \mathrm{~nm}$, which is comparable to the thickness of poly-Si film used.

\section{DYNAMIC PERFORMANCE}

Figure 6 shows the schematic drawing of the experimental setup. The fabricated IR detector is actuated by applying sinusoidal voltage $V_{\text {drive }}$ with the offset between the Si substrate. The amplitude of the driving voltage is less than $6 \mathrm{~V}$ applied to the poly-Si resonator. The ground is connected to the substrate. The red laser (wavelength: 650nm) for sensing the resonator movement is angularly irradiated onto the detector. The laser spot is aligned to the end one of arrayed resonators. The reflected and the diffracted light from the twisting resonator is monitored using a photodiode, whose signal response is measured using the frequency response analyzer (NF corp., FRA5097) with the reference of the driving signal. Since the gold has high reflectivity against IR, IR absorption and the thermal bending is small in the fabricated device. To generate bending of the resonator by the local 
heating, the blue laser (wavelength: $407 \mathrm{~nm}$ ) is used instead of IR. The gold is known to have the absorption of about $65 \%$ against the blue light[30]. The blue laser is irradiated through the microscope objective lens. The sample can be set in the vacuum chamber evacuated by the dry scroll pump having the window for allowing the optical access.

Figure 7 shows the frequency response of the fabricated resonator. Under the ambient temperature condition, the resonant frequency is observed at $32 \mathrm{kHz}$ as shown in Fig. 7(a). Q factor calculated from the resonant frequency ratio to the full peak width at $-3 \mathrm{~dB}$ level below the top is 2.5 . For decreasing the squeezed film damping, the measurements are conducted under the vacuum (1.6Pa). Figure 7(b) shows the response around the peak. The resonant frequency is at $32.6 \mathrm{kHz}$ and Q factor increases to 188 . For obtaining the frequency resolution in the resonator response, the narrower peak is advantageous. There are rooms for improving the resonator performance. When another resonator is driven at 1.0 and $2.0 \times 10^{-3} \mathrm{~Pa}$ using the turbo molecular pump, Q factor increases from 1300 to 4100 . The squeezed film damping seems to be remained in the data of Fig. 7 . The above improvement is also obtained by introducing the vapor HF etching in the fabrication process. The device described here may have the damage or the defect in the film. When the initial profile can be controlled to bend upward at the resonator center part, the resultant wider gap between the substrate will further decrease the squeezed film damping.

Figure 8 shows the resonant frequency as the function of the light intensity. The device used has the resonant frequency of $59 \mathrm{kHz}$ before the blue laser irradiation. The blue laser is focused on the IR resonator for heating. With increasing the laser power to $20 \mathrm{~kW} / \mathrm{m}^{2}$, the resonant frequency reaches to $64.6 \mathrm{kHz}$. The resonant frequency shift up to $10 \%$ of the initial value is confirmed. This corresponds to the increase of the spring constant by about $20 \%$. This experiment reflects simply the limit of the maximum power of the blue laser used. The further larger shift is considered to be possible.

In order to evaluate the sensitivity against the temperature, the dependence of the resonant frequency on the temperature difference $\Delta T$ is measured. The substrate temperature is changed keeping the blue laser irradiation. This will decrease the temperature difference $\Delta T$. Figure 9 shows 
the resonant frequency as the function of the substrate temperature increases. The resonant frequency decreases when $\Delta T$ decreases by increasing the substrate temperature. The decreasing rate can be evaluated to be about $30 \mathrm{~Hz} / \mathrm{K}$, which corresponds to the thermal coefficient against the resonant frequency of $830 \mathrm{ppm} / \mathrm{K}$. These values are almost same for ambient and vacuum conditions. Cabuz et al. reported the sensitivity of -11.2 and $15.7 \mathrm{~Hz} / \mathrm{K}$ for the bridge type bending resonator, which corresponds to the thermal coefficient against the resonant frequency of -370 and $410 \mathrm{ppm} / \mathrm{K}[17]$. Ono et al. reported $-12 \mathrm{~Hz} / \mathrm{K}$ for the cantilever type bending resonator with the parametrical amplifying, which corresponding to the thermal coefficient against the resonant frequency of -560 $\mathrm{ppm} / \mathrm{K}[18]$. The proposed resonator is promising to realize the further higher sensitivity than the values demonstrated here considering that the present resonator has the rooms for improving. When its profile has the initial bending upward not to be flat or downward, the nonlinear effect of the torsional spring will be larger giving the higher sensitivity.

\section{CONCLUSIONS}

New resonant IR detector which uses twisting motion is first demonstrated. The thin film torsion bar has the large nonlinearity and is advantageous for obtaining the higher sensitivity. The IR detector consists of two parts. One is the thin film torsion bar and another is bending center part. They can be basically designed separately. The center part has bi-layer structure to induce bending against the temperature increase caused by the IR absorption. This static bending hardens the spring constant of the torsion bar. The resonant frequency is observed to increase up to $10 \%$ of the original value without the unstable phenomenon when the fabricated IR detector is heated by the laser irradiation. The fabricated IR detector demonstrates the sensitivity of $30 \mathrm{~Hz} / \mathrm{K}$, which is larger than the values obtained from the previously reported Si based devices using the bending motion. The shifting ratio of $830 \mathrm{ppm} / \mathrm{K}$ against the resonant frequency is larger than devices including the high-frequency quartz and $\mathrm{ZnO}$ detectors. The device is promising and there are many rooms for the improvement (for example, initial stress control, Q factor, IR absorber). 


\section{ACKNOWLEDGEMENTS}

This research was supported by JSPS Grant-in-Aid for Challenging Exploratory Research (21656105), JST-project to develop innovative seeds (08-206), MEXT program for forming strategic research infrastructures for private universities from 2011. The authors give thanks to Mr. K. Miyake of Rexxam Co., Ltd. for the technical support on critical point drying. 


\section{Figure Captions}

Figure 1: (a) Schematic drawing of the IR detector using twisting motion. The electrostatic driving is used. Illustration of the moving element. (b) The initial condition for the twisting motion. (c) The bended condition with the twisting motion after local heating due to the IR absorption.

Figure 2: Analytical model of the thin film torsion bar for expressing the displacements relating with the twisting and the bending. The supposed design values relating the torsion bar are the width $w=5 \mu \mathrm{m}$, the thickness $t=400 \mathrm{~nm}$, the length $L=2 l=40 \mu \mathrm{m}$, the bending height $z_{m}=2 t$, the twisting angle $\theta_{\sigma}=5 \mathrm{deg}$, Young's modulus $E=153 \mathrm{GPa}$, the torsional rigidity $G=66.5 \mathrm{GPa}$, the film stress $\sigma_{0}=500 \mathrm{MPa}$.

Figure 3: Fabrication sequence showing AA' cross-section.

Figure 4: (a) One device array prepared using the stencil mask. (b) Another device array prepared with Au/Cr etching. (c) Magnified view around the torsion bar.

Figure 5: (a) Schematic drawing of the experimental setup for measuring the static bending of the IR detector. (b) Profiles of one IR detector bended by heating from the substrate side. The inserted top photo is for showing the position relative to the device structure ( $\mathrm{Au} / \mathrm{Cr}$ area, center part, torsion bar).

Figure 6: Schematic drawing of the experimental setup for measuring the dynamic response of the resonant IR detector.

Figure 7: Frequency responses of the resonant IR detector under (a) ambient and (b) vacuum conditions. The relatively noisy plot for (b) can be attributed to the higher Q value, which needs the time for scanning the frequency.

Figure 8: Resonant frequency as a function of the incident laser power (not the absorbed power).

Figure 9: Resonant frequency change as a function of the substrate temperature. 


\section{REFERENCES}

[1] A. M. Itsuno, J. D. Phillips, S. Velicu, "Mid-wave infrared HgCdTe nBn photodetector", Appl. Phys. Lett., Vol. 100 (2012) 161102.

[2] H. W. Li, B. E. Kardynał, P. See, A. J. Shields, P. Simmons, H. E. Beere, D. A. Ritchie, "Quantum dot resonant tunneling diode for telecommunication wavelength single photon detection”, Appl. Phys. Lett., Vol. 91, 2007, 073516.

[3] M. W. Knight, H. Sobhani, P. Nordlander, N. J. Halas, "Photodetection with Active Optical Antennas", Science 6 May 2011: Vol. 332 no. 6030 pp. 702-704.

[4] M. Kimata "Comprehensive Microsystems - Fundamentals,technology and applications-" Elsevier Science Ltd. (2007.12.24) Volume Three, 3.04 IR Imaging, pp.113-163.

[5] B. E. Cole, R. E. Higashi, R. A. Wood, "Monolithic Two-Dimensional Arrays of Micromachined Microstructures for Infrared Applications” Proc. IEEE, Vol. 86 (1998) pp.1679-1686.

[6] H. Wu, S. Grabarnik, A. Emadi, G. de Graaf, R. F. Wolffenbuttel, “A thermopile detector array with scaled TE elements for use in an integrated IR microspectrometer”, J. Micromech. Microeng. 18 (2008) 064017.

[7] D. Xu, B. Xiong, Y. Wang, "Design, fabrication and characterization of a front-etched micromachined thermopile for IR detection”, J. Micromech. Microeng. 20 (2010) 115004.

[8] H. Wu, A. Emadi, P. M. Sarro, G. de Graaf, R. F. Wolffenbuttel, “A surface micromachined thermopile detector array with an interference-based absorber”, J. Micromech. Microeng. 21 (2011) 074009.

[9] H. Zhou, P Kropelnicki, J. M. Tsai, C. Lee, "Development of a thermopile infrared sensor using stacked double polycrystalline silicon layers based on the CMOS process", J. Micromech. Microeng. 23 (2013) 065026.

[10] Z. Djuric, D. Randjelovic, I. Jokic, J. Matovic, J. Lamovec, “A new approach to IR bimaterial detectors theory", Infrared Physics \& Technology 50 (2007) 51-57. 
[11] D. Fengliang, Z. Qingchuan, C. Dapeng, P. Liang, G. Zheying, W. Weibing, D. Zhihui, W. Xiaoping, “An uncooled optically readable infrared imaging detector”, Sens. Act. A 133 (2007) $236-242$.

[12] S. Huang, H. Tao, I-K. Lin, X. Zhang, "Development of double-cantilever infrared detectors: Fabrication, curvature control and demonstration of thermal detection", Sens. Act. A 145-146 (2008) 231-240.

[13] I. W. Kwon, J. E. Kim, C. H. Hwang, Y. S. Lee, H. C. Lee, "Design and fabrication of a capacitive infrared detector with a floating electrode and thermally isolatable bimorph legs", Sens. Act. A 147 (2008) 391-400.

[14] J. Gao, Q. Zhang, B. Jiao, D. Chen, “Optical sensitivity analysis of a bent micro reflector array in uncooled infrared imaging”, J. Micromech. Microeng. 19 (2009) 095018.

[15] H. Kwon, K. Suzuki, K. Ishii, H. Yagi, H. Honda, M. Atsuta, I. Fujiwara, K. Sasaki, M. Ogata, R. Ueno, H. Funaki, “A SOI-based CMOS-MEMS IR image sensor with partially released reference pixels”, J. Micromech. Microeng. 21 (2011) 025028.

[16] W. Jiang, W. Ou, A. Ming, Z. Liu, X. Zhang, “Design and analysis of a high fill-factor SOI diode uncooled infrared focal plane array”, J. Micromech. Microeng. 23 (2013) 065004.

[17] C. Cabuz, S. Shoji, K. Fukatsu, E. Cabuz, K. Minami, M. Esashi, "Fabrication and packaging of a resonant infrared sensor integrated in silicon”, Sens. Act. A, 43 (1994) 92-99.

[18] T. Ono, H. Wakamatsu, M. Esashi, "Parametrically amplified thermal resonant sensor with psudo-cooling effect”, J. Micromech. Microeng., Vol. 15, 2005, pp. 2282-2288.

[19] K. Isamoto, K. Kato, A. Morosawa, C. Chong, H. Fujita, H. Toshiyoshi, ”A 5-V operated MEMS variable optical attenuator by SOI bulk micromachining”, IEEE J. Selected Topics in Quantum Electron., vol. 10 (2004) pp.570-578.

[20] A. Miner, V. Milanovic, "High temperature operation of gimbal-less two axis micromirrors", Proc. IEEE/LEOS Int. Conf. on Opt. MEMS \& Nanophotonics, TuP19 (2007) pp.91-92.

[21] M. Sasaki, M. Fujishima, K. Hane, H. Miura, “Simultaneous Realization of Stabilized 
Temperature Characteristics and Low-Voltage Driving of Micromirror Using Thin Film Torsion Bar of Tensile Poly-Si”, IEEE J. Selected Topics in Quantum Electron., Vol. 15 (2009) pp.1455-1462.

[22] P. Kao, S. Tadigadapa, "Micromachined quartz resonator based infrared detector array", Sens. Act. A 149 (2009) 189-192.

[23] Z. Wang, X. Qiu, S. Jui Chen, W. Pang, H. Zhang, J. Shi, H. Yu, “ZnO based film bulk acoustic resonator as infrared sensor", Thin Solid Films 519 (2011) 6144-6147.

[24] M. Sasaki, S. Yuki, K. Hane, "Performance of Tense Thin-Film Torsion Bar for Large Rotation and Low-Voltage Driving of Micromirror”, IEEE J. Sel. Topics Quantum Electron., vol. 13 (2007) pp.290-296.

[25] S. K. Kundu, S. Ogawa, S. Kumagai, M. Fujishima, K. Hane, M. Sasaki, "Nonlinear Spring Effect of Tense Thin-Film Torsion Bar Combined with Electrostatic Driving”, Sens. Act. A, Vol. 195 (2013) pp.83-89.

[26] S. D. Senturia, "Microsystem Design”, Kluwer Academic Publishers (2001) Chap. 10, Section 10.4 .

[27] H. Miura, H. Ohta, N. Okamoto, T. Kaga, "Crystallization-induced stress in silicon thin films", Appl. Phys . Lett., Vol. 60 (1992) pp. 2746-2748.

[28] Allen Cowen, Busbee Hardy, Ramaswamy Mahadevan, Steve Wilcenski, “PolyMUMPs Design Handbook", MEMSCAP Inc. http://www.memscap.com/_data/assets/pdf_file/0019/1729/ PolyMUMPs-DR-13-0.pdf.

[29] G. Lammel, S. Schweizer, P. Renaud, "Optical microscanners and microspectrometers using thermal bimorph actuators", Kluwer Academic Publishers, Chapter 2.

[30] E. Hecht, “Optics”, Pearson Education, Inc., Chapter 4. 


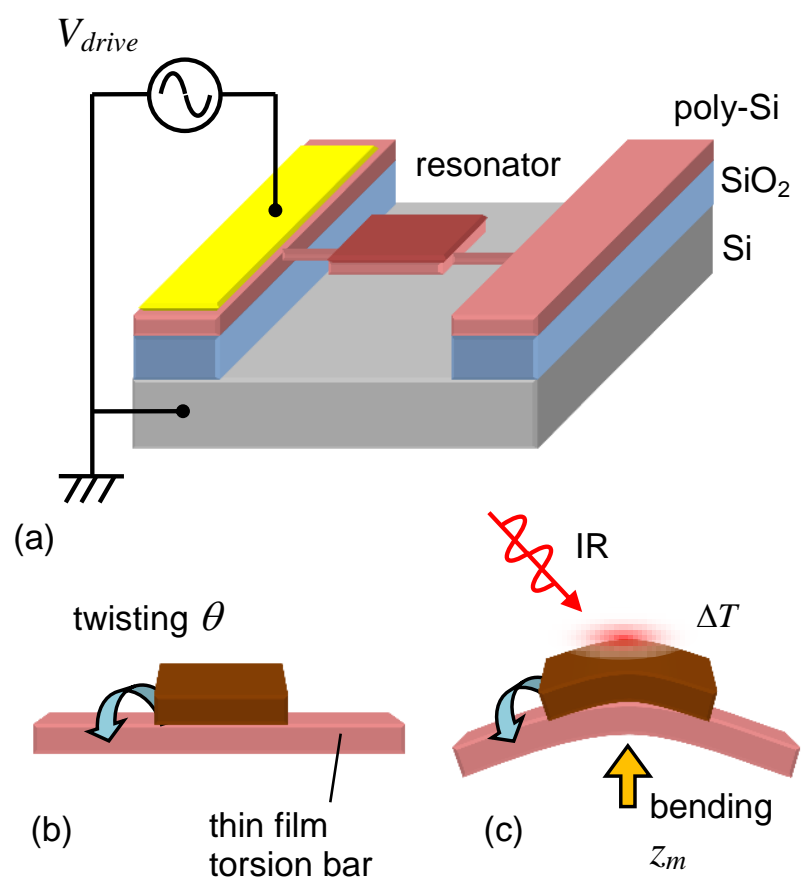

Figure 1: (a) Schematic drawing of the IR detector using twisting motion. The electrostatic driving is used. Illustration of the moving element. (b) The initial condition for the twisting motion. (c) The bended condition with the twisting motion after local heating due to the IR absorption. 


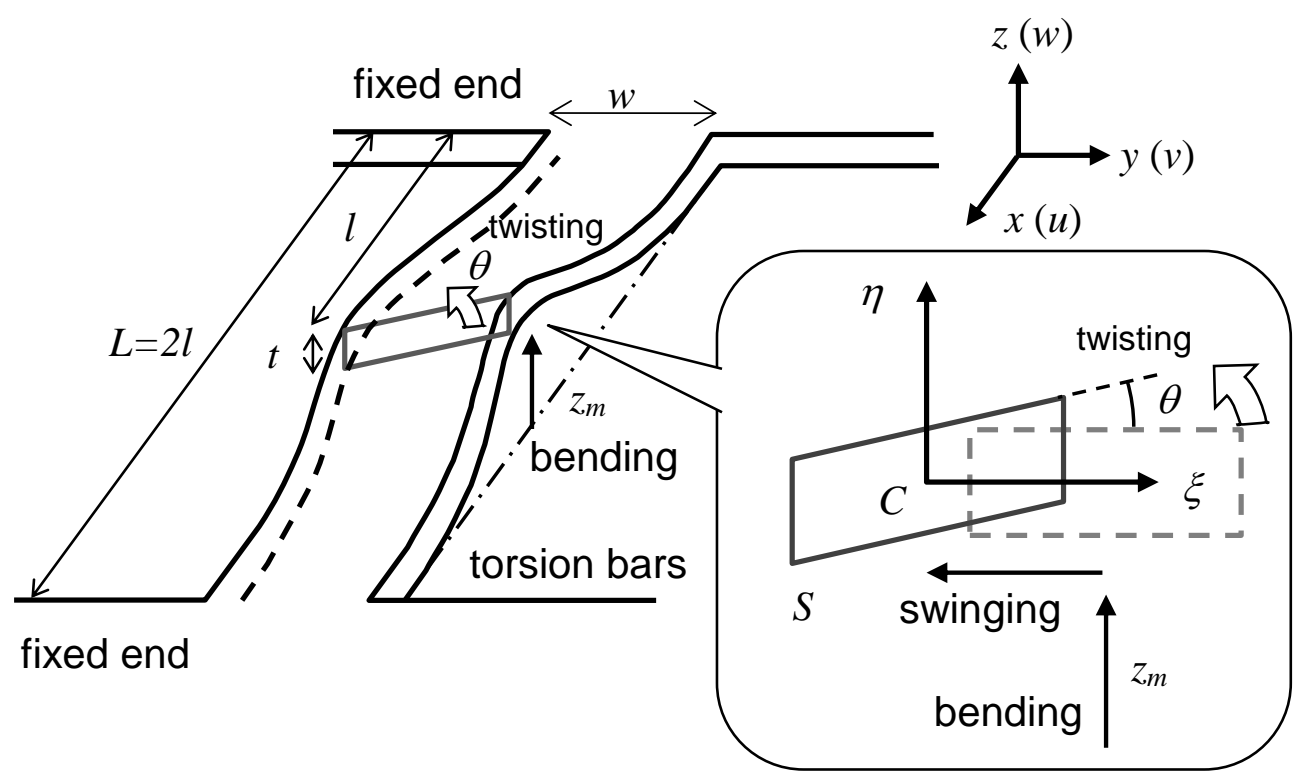

Figure 2: Analytical model of the thin film torsion bar for expressing the displacements relating with the twisting and the bending. The supposed design values relating the torsion bar are the width $w=5 \mu \mathrm{m}$, the thickness $t=400 \mathrm{~nm}$, the length $L=2 l=40 \mu \mathrm{m}$, the bending height $z_{m}=2 t$, the twisting angle $\theta_{0}=5 \mathrm{deg}$, Young's modulus $E=153 \mathrm{GPa}$, the torsional rigidity $G=66.5 \mathrm{GPa}$, the film stress $\sigma_{0}=500 \mathrm{MPa}$. 

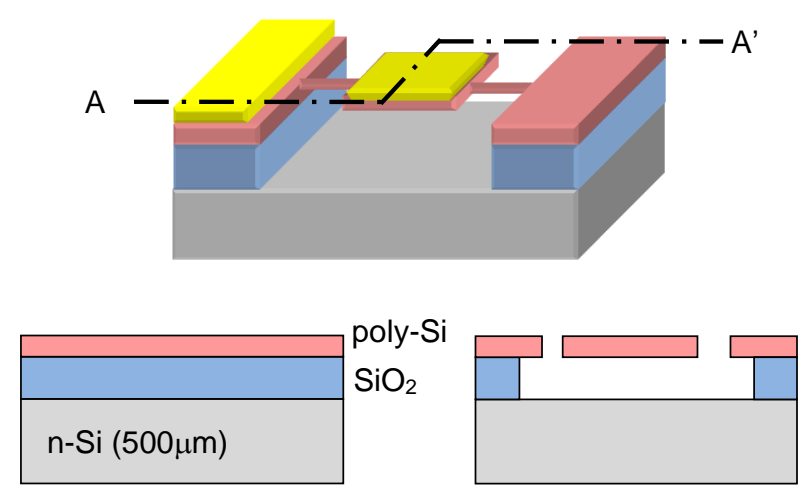

(1) a-Si depo. B ion implantation, anneal

(4) $\mathrm{SiO}_{2}$ etching

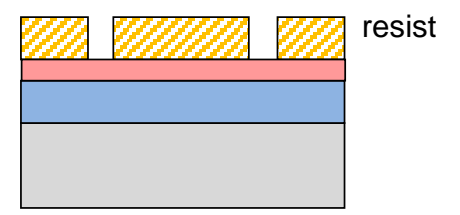

(2) patterning

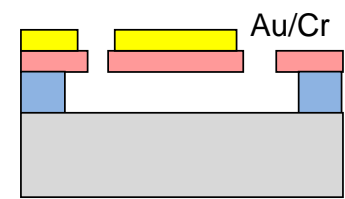

(5) Au/Cr depo.

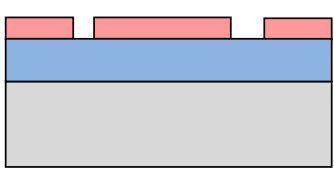

(3) poly-Si etching

Figure 3: Fabrication sequence showing AA' cross-section. 

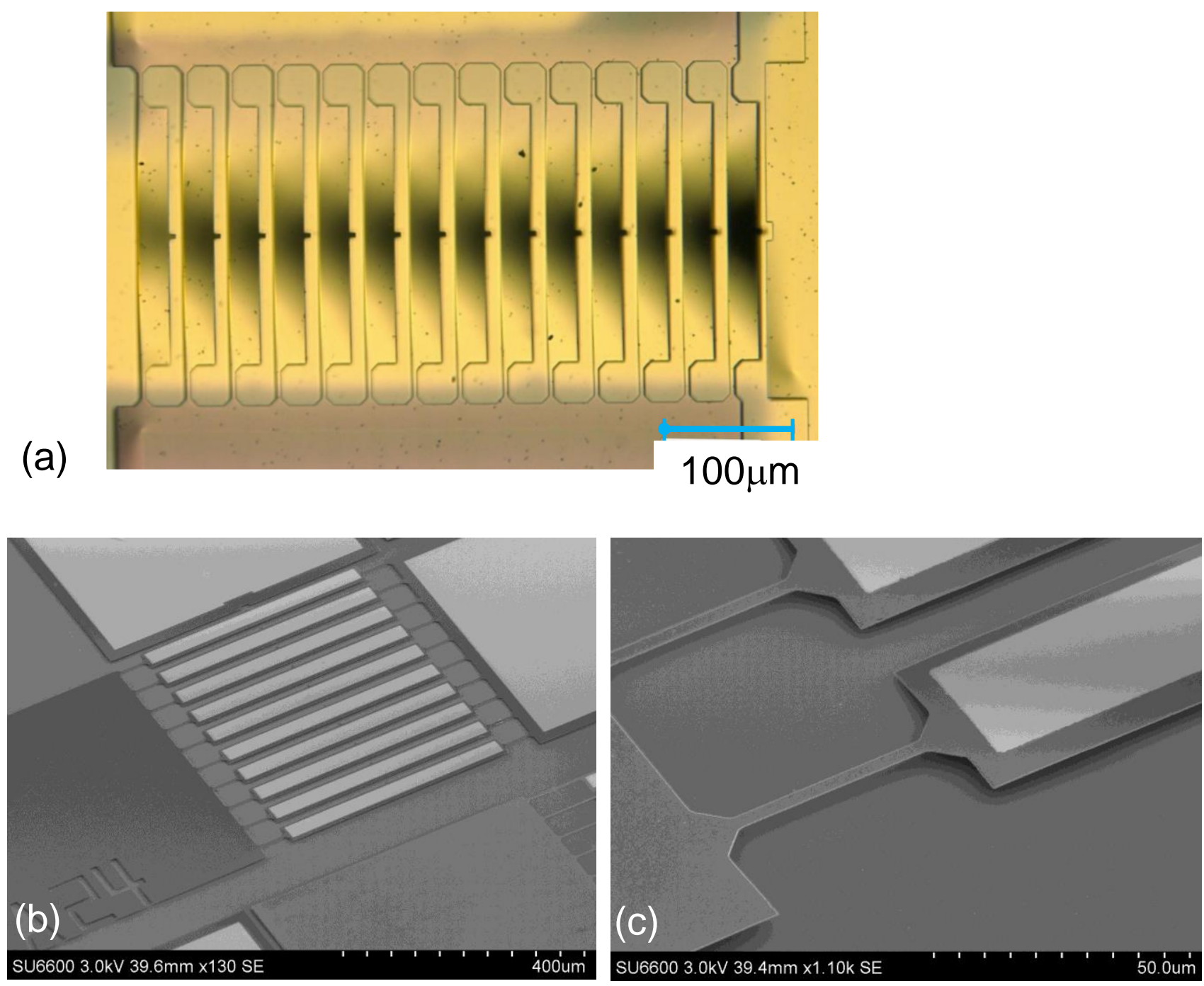

Figure 4: (a) One device array prepared using the stencil mask. (b) Another device array prepared with $\mathrm{Au} / \mathrm{Cr}$ etching. (c) Magnified view around the torsion bar. 

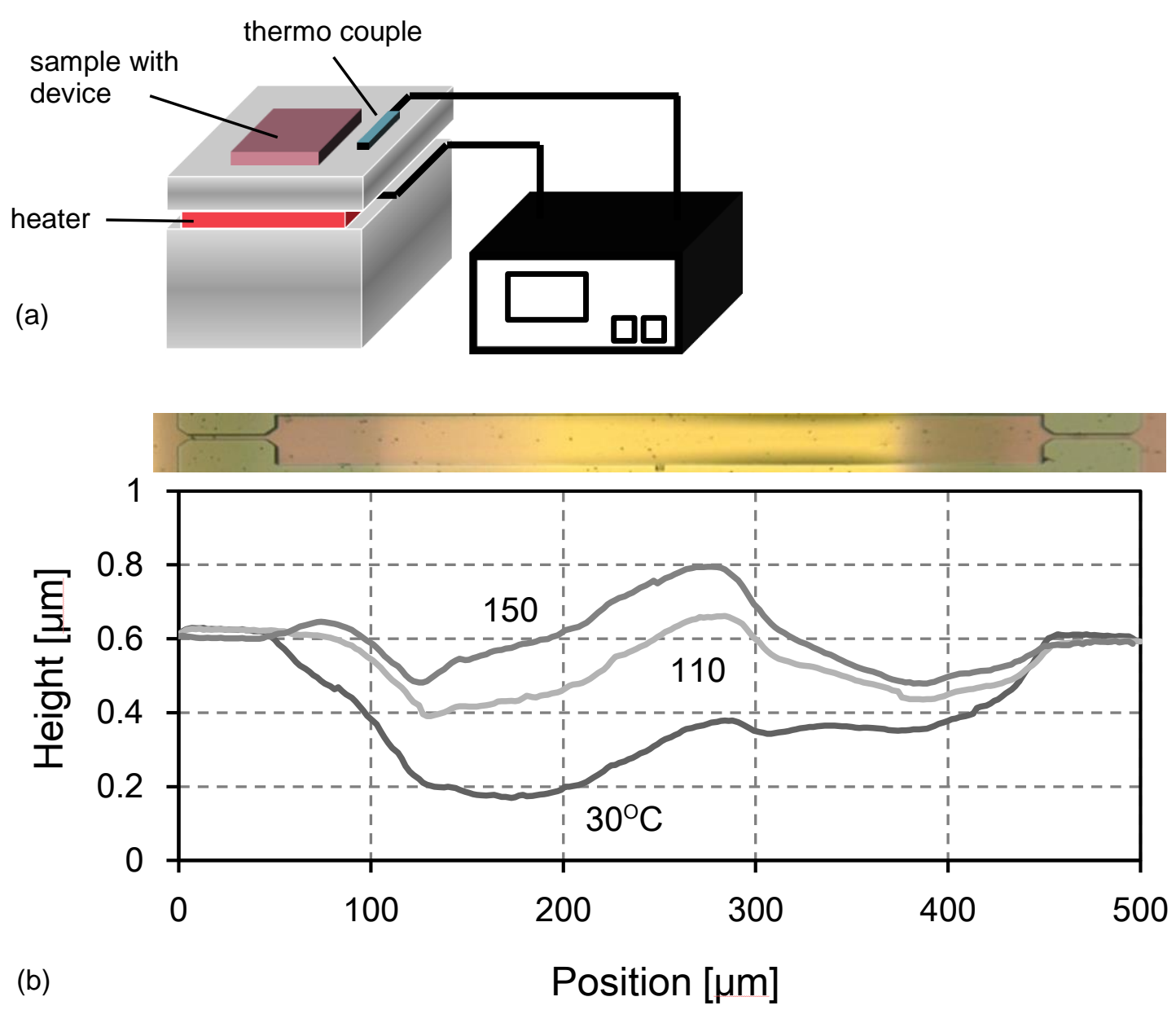

Figure 5: (a) Schematic drawing of the experimental setup for measuring the static bending of the IR detector. (b) Profiles of one IR detector bended by heating from the substrate side. The inserted top photo is for showing the position relative to the device structure ( $\mathrm{Au} / \mathrm{Cr}$ area, center part, torsion bar). 


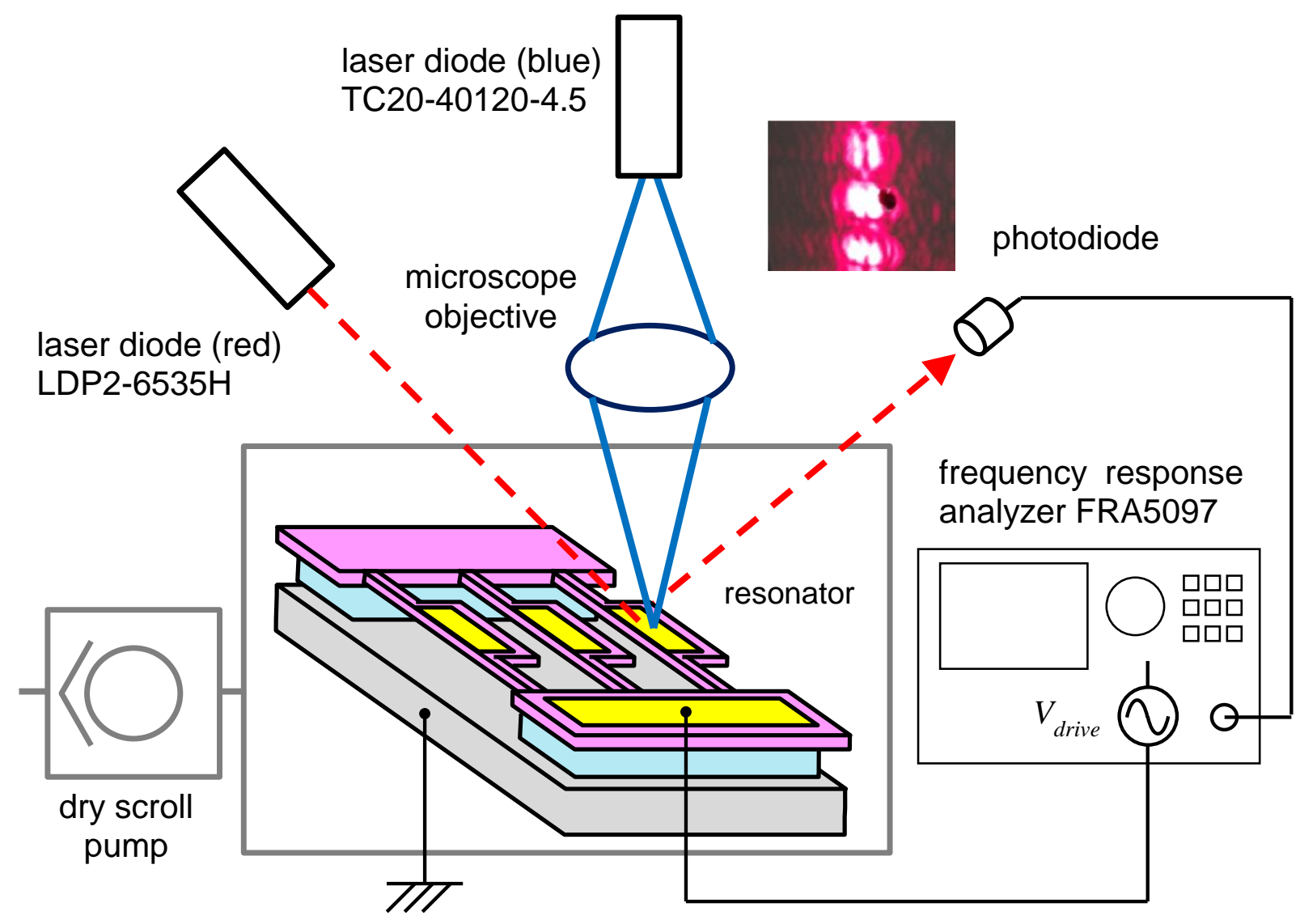

Figure 6: Schematic drawing of the experimental setup for measuring the dynamic response of the resonant IR detector. 

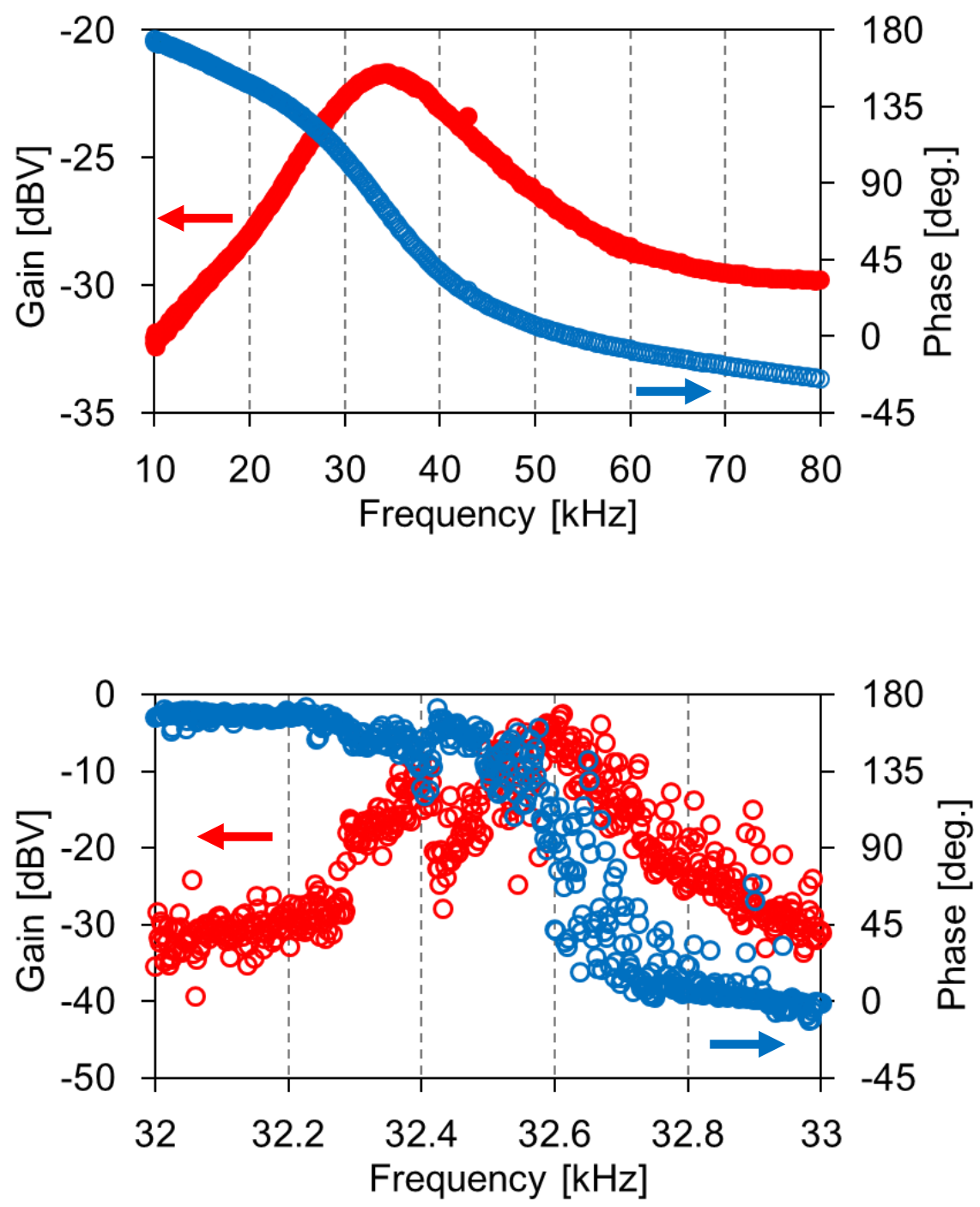

(b)

Figure 7: Frequency responses of the resonant IR detector under (a) ambient and (b) vacuum conditions. The relatively noisy plot for (b) can be attributed to the higher Q value, which needs the time for scanning the frequency. 


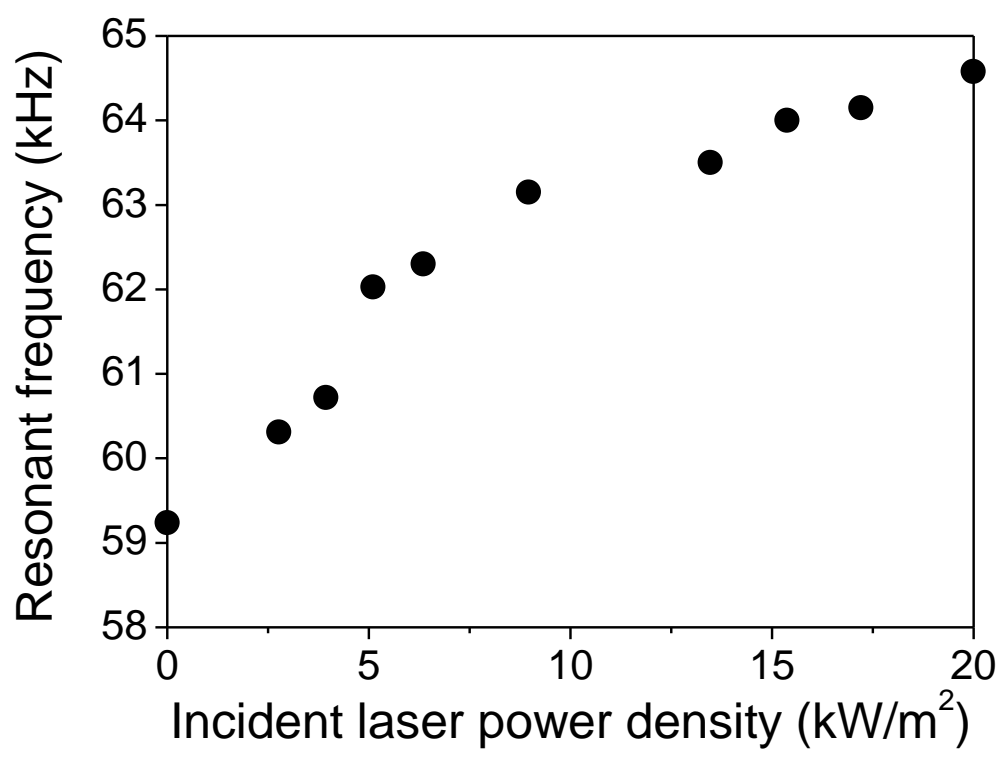

Figure 8: Resonant frequency as a function of the incident laser power (not the absorbed power). 


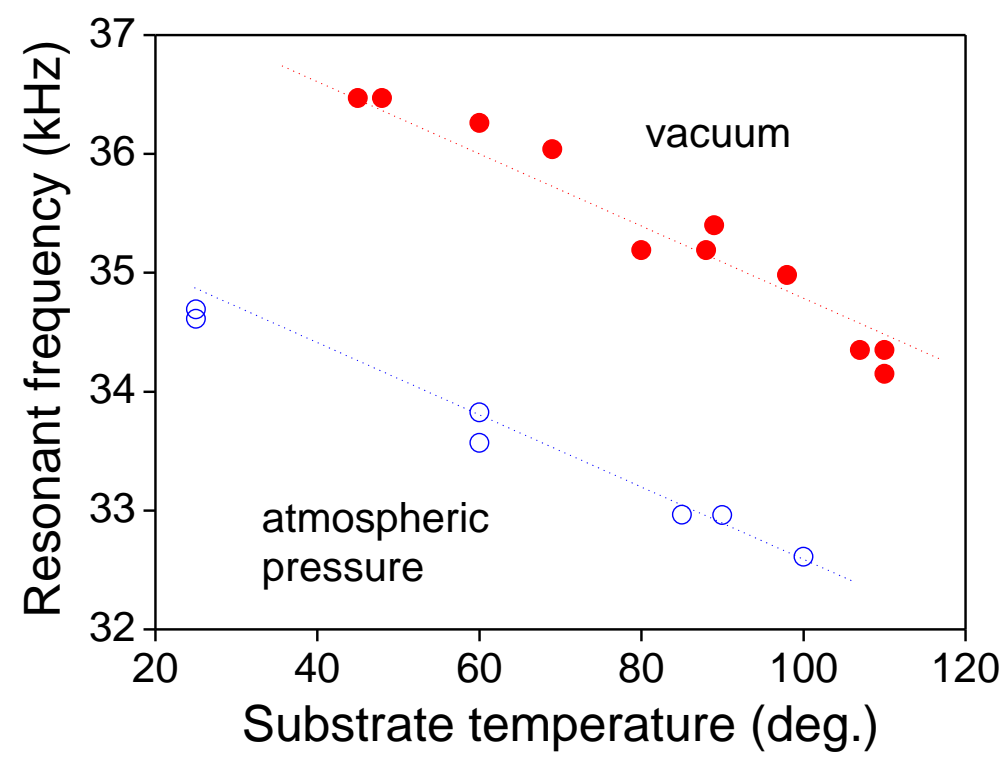

Figure 9: Resonant frequency change as a function of the substrate temperature. 